\title{
OSTEOARTHRITIS
}

\section{Subchondral bone erosion in hand $O A$ : insights into the role of inflammation}

Erosive hand osteoarthritis (EOA) is a subset of hand osteoarthritis (HOA) in which subchondral erosions are seen on radiographs of the interphalangeal joints (IPJs). EOA is associated with a higher clinical burden than nonerosive disease. The pathogenesis of EOA is not well understood, but inflammation is thought to be involved. This theory, together with their previous work suggesting an underlying systemic cause for erosion development, led Marion Kortekaas et al. to perform a study in which they show that more inflammatory signs are present in joints from patients with EOA than those with non-EOA, even in joints that are nonerosive.

Consecutive patients $(n=64)$ being seen at a rheumatology outpatient clinic at the Leiden University Medical Centre who met the American College of Rheumatology criteria for HOA were recruited, and 55 of these were included in this cross-sectional study. The Verbruggen-Veys anatomical phase score was used to assess radiographs of all 18 IPJs, and ultrasonography was used to assess joint effusion, synovial thickness and power Doppler signal (PDS; a measurement of vascularization, which in a joint indicates active inflammation).

$51 \%$ of patients had EOA, as defined in this study by the presence of at least one erosive joint. Synovial thickening, effusion and PDS were seen in 13\%, $50 \%$ and $15 \%$, respectively, of 94 erosive joints, and in $10 \%, 26 \%$ and $8 \%$ of 896 nonerosive joints (from 28 patients with EOA and 27 patients with non-EOA). Effusion and PDS (summated scores and the number of affected joints) were higher in patients with EOA than those with non-EOA; the summated scores for synovial thickening were higher in EOA than in non-EOA but the number of joints with synovial thickening did not differ between the two groups.

Nonerosive IPJs from patients with EOA had more PDS and effusion (i.e. more inflammation) than those from patients with non-EOA even though these joints were not erosive. "We found differences in inflammatory features between EOA and non-EOA," explains Kortekaas. "Since nonerosive joints in EOA showed more inflammatory signs, this suggests that a systemic process is taking place and could be supportive of the idea that EOA is a different subset of HOA associated with more inflammation."

Jenny Buckland

Original article Kortekaas, M. C. et al. In erosive hand osteoarthritis more inflammatory signs on ultrasound are found than in the rest of hand osteoarthritis. Ann. Rheum. Dis. doi:10.1136/annrheumdis-2012-201458 